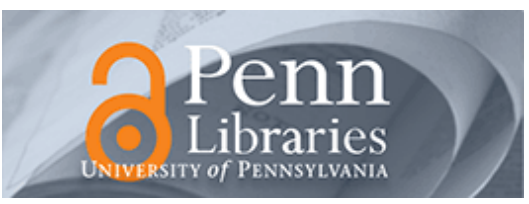

University of Pennsylvania

ScholarlyCommons

October 2006

\title{
Sensor Data Fusion for Body State Estimation in a Hexapod Robot With Dynamical Gaits
}

\author{
Pei-Chun Lin \\ University of Pennsylvania \\ Haldun Komsuoglu \\ University of Pennsylvania \\ Daniel E. Koditschek \\ University of Pennsylvania, kod@seas.upenn.edu
}

Follow this and additional works at: https://repository.upenn.edu/ese_papers

\section{Recommended Citation \\ Pei-Chun Lin, Haldun Komsuoglu, and Daniel E. Koditschek, "Sensor Data Fusion for Body State Estimation in a Hexapod Robot With Dynamical Gaits", . October 2006.}

Copyright IEEE 2006. Reprinted from IEEE Transactions on Robotics, Volume 22, Issue 5, October 2006, pages 932-943.

This material is posted here with permission of the IEEE. Such permission of the IEEE does not in any way imply IEEE endorsement of any of the University of Pennsylvania's products or services. Internal or personal use of this material is permitted. However, permission to reprint/republish this material for advertising or promotional purposes or for creating new collective works for resale or redistribution must be obtained from the IEEE by writing to pubs-permissions@ieee.org. By choosing to view this document, you agree to all provisions of the copyright laws protecting it.

This paper is posted at ScholarlyCommons. https://repository.upenn.edu/ese_papers/202

For more information, please contact repository@pobox.upenn.edu. 


\title{
Sensor Data Fusion for Body State Estimation in a Hexapod Robot With Dynamical Gaits
}

\begin{abstract}
We report on a hybrid 12-dimensional full body state estimator for a hexapod robot executing a jogging gait in steady state on level terrain with regularly alternating ground contact and aerial phases of motion. We use a repeating sequence of continuous time dynamical models that are switched in and out of an extended Kalman filter to fuse measurements from a novel leg pose sensor and inertial sensors. Our inertial measurement unit supplements the traditionally paired three-axis rate gyro and three-axis accelerometer with a set of three additional three-axis accelerometer suites, thereby providing additional angular acceleration measurement, avoiding the need for localization of the accelerometer at the center of mass on the robot's body, and simplifying installation and calibration. We implement this estimation procedure offline, using data extracted from numerous repeated runs of the hexapod robot RHex (bearing the appropriate sensor suite) and evaluate its performance with reference to a visual ground-truth measurement system, comparing as well the relative performance of different fusion approaches implemented via different model sequences.
\end{abstract}

\section{Keywords}

extended kalman filter (ekf), hybrid estimation model, inertial measurement unit (imu), legged robot, leg pose sensor (Ips), sensor fusion

\section{Comments}

Copyright IEEE 2006. Reprinted from IEEE Transactions on Robotics, Volume 22, Issue 5, October 2006, pages 932-943.

This material is posted here with permission of the IEEE. Such permission of the IEEE does not in any way imply IEEE endorsement of any of the University of Pennsylvania's products or services. Internal or personal use of this material is permitted. However, permission to reprint/republish this material for advertising or promotional purposes or for creating new collective works for resale or redistribution must be obtained from the IEEE by writing to pubs-permissions@ieee.org. By choosing to view this document, you agree to all provisions of the copyright laws protecting it. 


\title{
Sensor Data Fusion for Body State Estimation in a Hexapod Robot With Dynamical Gaits
}

\author{
Pei-Chun Lin, Member, IEEE, Haldun Komsuoḡlu, Member, IEEE, and Daniel E. Koditschek, Fellow, IEEE
}

\begin{abstract}
We report on a hybrid 12-dimensional full body state estimator for a hexapod robot executing a jogging gait in steady state on level terrain with regularly alternating ground contact and aerial phases of motion. We use a repeating sequence of continuous time dynamical models that are switched in and out of an extended Kalman filter to fuse measurements from a novel leg pose sensor and inertial sensors. Our inertial measurement unit supplements the traditionally paired three-axis rate gyro and three-axis accelerometer with a set of three additional three-axis accelerometer suites, thereby providing additional angular acceleration measurement, avoiding the need for localization of the accelerometer at the center of mass on the robot's body, and simplifying installation and calibration. We implement this estimation procedure offline, using data extracted from numerous repeated runs of the hexapod robot RHex (bearing the appropriate sensor suite) and evaluate its performance with reference to a visual ground-truth measurement system, comparing as well the relative performance of different fusion approaches implemented via different model sequences.
\end{abstract}

Index Terms-Extended Kalman filter (EKF), hybrid estimation model, inertial measurement unit (IMU), legged robot, leg pose sensor (LPS), sensor fusion.

\section{INTRODUCTION}

T HE hexapod RHex [1] exhibits unprecedented mobility for a legged autonomous robot [2]. Using an open loop feedforward control strategy, the machine runs at speeds exceeding five body lengths per second on even terrain [3], and negotiates badly broken and unstable surfaces, as well as stairs [4], [5]. Initial empirical studies of controllers relying on cheap and inaccurate sensory feedback cues have resulted in significantly improved performance (inclinometers on slopes [6]; leg touchdown cues over broken terrain [7]) and entirely new behaviors (body-pitch-sensitive accelerometers for flips [8] and bipedal gaits [9]; leg touchdown cues for pronking gaits [10]). Theoretical considerations and simulation evidence [11] suggest that the availability of accurate, full body state estimates, as well as force interactions with the surrounding environment throughout the stance and aerial phases of locomotion, should confer considerably greater agility still.

However, building a sensor suite that can deliver full body state information-six configuration coordinates together with

Manuscript received June 21, 2005; revised February 21, 2006. This paper was recommended by Associate Editor G. Sukhatme and Editor H. Arai upon evaluation of the reviewers' comments. This work was supported by DARPA/SPAWAR under Contract N66001-00-C-8026 and under Contract N66001-03-C-8045. This paper was presented in part at the IEEE International Conference on Robotics and Automation, Barcelona, Spain, April 2005.

The authors are with the Department of Electrical and Systems Engineering, University of Pennsylvania, Philadelphia, PA 19104 USA (e-mail: peichun@seas.upenn.edu; haldunk@seas.upenn.edu; kod@ese.upenn.edu).

Digital Object Identifier 10.1109/TRO.2006.878954

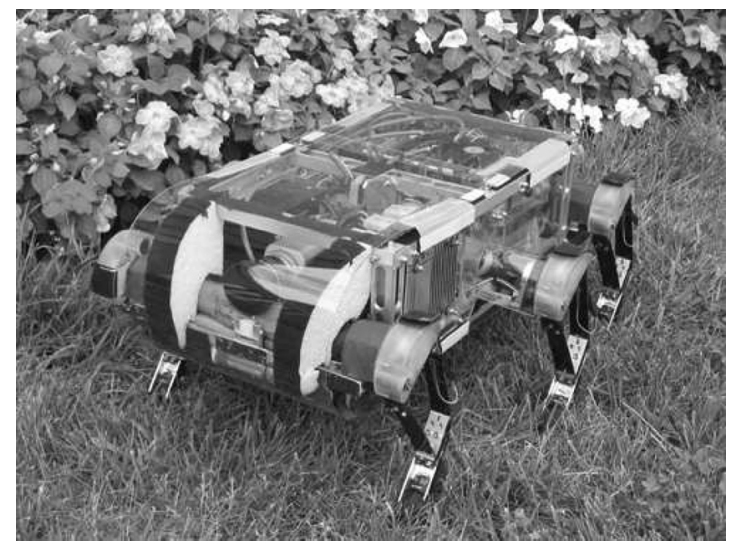

Fig. 1. RHex with 4-bar compliant legs equipped with strain-based leg configuration sensors (LPS) stands on the meadow.

their six time derivatives-at data rates relevant to motor control $(\sim 1 \mathrm{kHz})$ remains a challenging problem in legged robotics because of the limitation of onboard instrumentation combined with extreme variations in operating regime. The traditional inertial measurement unit (IMU) for rigid bodies in flight typically lies out of the range of robotics applications because of its cost and excessive volume. Appropriately low-cost and small IMU packages typically suffer severe drift and saturation/sensitivity problems. Moreover, while ballistic flight models are quite accurate, legged machines by definition spend a large fraction of their locomotion duty cycle in ground contact. Therefore, the determination of an appropriate model is greatly complicated by the uncertainty in ground conditions (local terrain shape, slipperiness, and damping and compliance properties) and leg contact conditions (which legs are in stance).

Recently, we introduced a novel leg-strain-based full body pose estimator [hereafter referred to as the leg pose sensor (LPS)] for a hexapod robot in tripod stance ${ }^{1}$ [12] with practical implementation on RHex, pictured in Fig. 1. In that paper, we demonstrated that a memoryless transformation built from (data-driven phenomenological) models relating leg strain to configuration, coupled with a conventional kinematic model of leg configuration to body pose, can accurately estimate body position and orientation when the robot's three noncollinear legs are fixed on the ground (i.e., in each tripod stance). In walking gaits with no aerial phase, a complete 6-degree-of-freedom (DOF) body pose in continuous time can easily be extended from the above tripod-stance body pose, in principle, from a purely kinematic model without velocity state estimation [12]. In contrast, an alternating tripod runner

\footnotetext{
${ }^{1}$ This term denotes the mode of leg contact wherein the three toes of the front and rear ipsilateral legs and the middle contralateral leg of a tripod are all in contact with the ground.
} 
experiencing significant aerial phases (with the concomitant touchdown/liftoff transients) ${ }^{2}$ would seem to require full body state estimation, both velocity and configuration information. In order to build the required estimators, of course, the sensor suite must incorporate enough information to allow the reconstruction of full state from the record of past measurement filled in by some dynamical model.

During stance, complete 12-DOF continuous-time body state estimates can be computed from the LPS by means of direct measurement and recourse to online differentiation. Absent any other available sensors, these stance state estimates may be carried through the transient and flight phases only by the adoption of some dynamical prediction model. Although the LPS delivers accurate high-bandwidth body pose estimates during stance (potentially marred by drift effects resulting from toe slippage [12]), overall performance throughout a complete stride is limited by inaccuracies in the transient phase models and the deleterious effects of online differentiation [14]. In contrast, an IMU continuously delivers derivative (typically translational/linear acceleration and rotational/angular velocity) information over all phases of a stride. Saturation and drift effects in the physical sensor, however, can dramatically reduce the accuracy of the resulting integrated position estimates. The complementary strengths and weaknesses of the LPS and IMU promise a better body state estimate than either, alone, could achieve. In this paper, we demonstrate that that promise can be achieved.

The traditional IMU (TIMU), comprised of three accelerometers (for linear acceleration) and three gyros (for angular velocity), can readily provide full 12 -DOF body state estimates when it is precisely located, carefully calibrated, and its output filtered appropriately. Unlike the translational state components, whose estimates require double integration of the accelerometer data, the rotational component estimates, requiring only a single integration step, might be imagined to incur smaller errors. However, these available sensory sources do not well subserve estimation models that take second-order dynamics into account. In the absence of angular acceleration measurements, the literature reveals a strong predilection for first-order dynamics [15] (usually assuming constant velocity), in preference to the direct differentiation that would be otherwise required without very accurate (and formally observable) dynamical models. Moreover, the general attitude toward contemporary low-cost microelectromechanical system (MEMS) gyros suggests that their inferior drift and saturation properties relative to MEMS accelerometers may vitiate any advantage at the signal processing stage. The considerable effort required to calibrate gyros ${ }^{3}$ detracts further from their inclusion in an IMU. These facts all suggest the utility of angular acceleration

\footnotetext{
${ }^{2}$ Note that hexapedal running gaits need not entail an aerial phase to be "dynamical" in the sense of requiring careful management of kinetic energy to insure balance and steady progress [13]. However, RHex develops its greatest energy efficiency and highest speeds in gaits with long aerial phases; hence, in this paper, we focus our empirical tests on a "jogging" gait with an aerial phase exceeding $25 \%$ of the complete stride. By "touchdown" and "liftoff" transients, we refer to intermediate configurations where some number of legs fewer than three are in ground contact.

${ }^{3}$ Acquiring for these low-cost MEMS devices the necessary gyro calibration data (the map from raw sensor output to angular rate with temperature compensation) generally provided by high-end commercial gyros requires a variable-speed-controlled turntable with temperature adjustment capability.
}

data and angular velocity data, if possible, from MEMS accelerometers for fusion. These considerations motivated King's [16] introduction of a novel nine-axis accelerometer suite, designed to measure angular acceleration and possibly angular velocity with no need for accompanying gyros. Unfortunately, this scheme requires that the accelerometer suite be very accurately installed in a specific spatial configuration. The falling cost and volume of MEMS-based accelerometers, together with the possibility of eliminating entirely such (theoretically innocent, but pragmatically onerous) installation requirements [16] motivates our introduction of a new 12-axis accelerometer suite - an advanced IMU (AIMU) capable of delivering linear/angular acceleration and angular velocity. We will show in Section III-B and Appendix I that the 12-axis accelerometer suite is theoretically capable of estimating all three aspects of body state with no recourse to gyros, but is impractical in our setting as a result of numerical ill-conditioning dependent upon the small baseline RHex's body affords. Therefore, along with the traditional 3-axis rate gyro, this 12 -axis accelerometer comprises the AIMU that we join in this paper to our previous LPS.

Developing an effective approach to modeling a legged robot whose running gait is to be stabilized by state feedback estimates raises the prospect of entering upon a circular path with no clear starting point. For an $n$-legged machine, there are $3^{n}$ possible formal Lagrangian models: touchdown-stick, touchdown-slip, and liftoff on each leg. These models include kinematic and dynamic properties of the legs whose small relative mass lends them at best negligible influence upon the body, apart from the actuators' effects. Hence, the adoption of an appropriately abstracted (12-dimensional or lower) family of approximate models has strong appeal. Moreover, the approach to gait stabilization that we favor provides growing theoretical [11] and empirical [8] justification for the validity of these lower-dimensional "template" control models. These abstracted models apply to the steady-state conditions that emerge from well-regulated gaits. On the one hand, tractable feedback controllers that restore these gaits rely upon accurate estimates; on the other hand, tractable filters based upon familiar dynamical models can be expected to yield accurate estimates only within well-controlled gaits. For the purposes of intelligent sensor development, we rely upon open-loop stabilizing gaits developed by offline tuning experiments [3] to bring the robot to a reliable steady-state condition, wherein familiar, tractable models can be readily implemented. Having established and verified the efficacy of these procedures for extracting reliable state estimates from the available sensor suite, future research will address the need for and possibility of introducing more accurate physical models, as well as switching them in and out as the leg contact conditions change during transients.

The idea of sensor fusion has spread widely within the mobile robotics community, largely for application to wheeled vehicles, addressing algorithm development [17], controller design [18], and some practical implementation. This literature includes navigation systems equipped with IMU alone using Kalman filtering [15], IMU/GPS fusion, sensor fault detection [19], model selection [20], and IMU/vision fusion [21]. However, for legged robots, we have found only a very few accounts of sensor fusion, addressing such problems as sonar-based localization [22] and multilayered-decision algorithms [23]. 


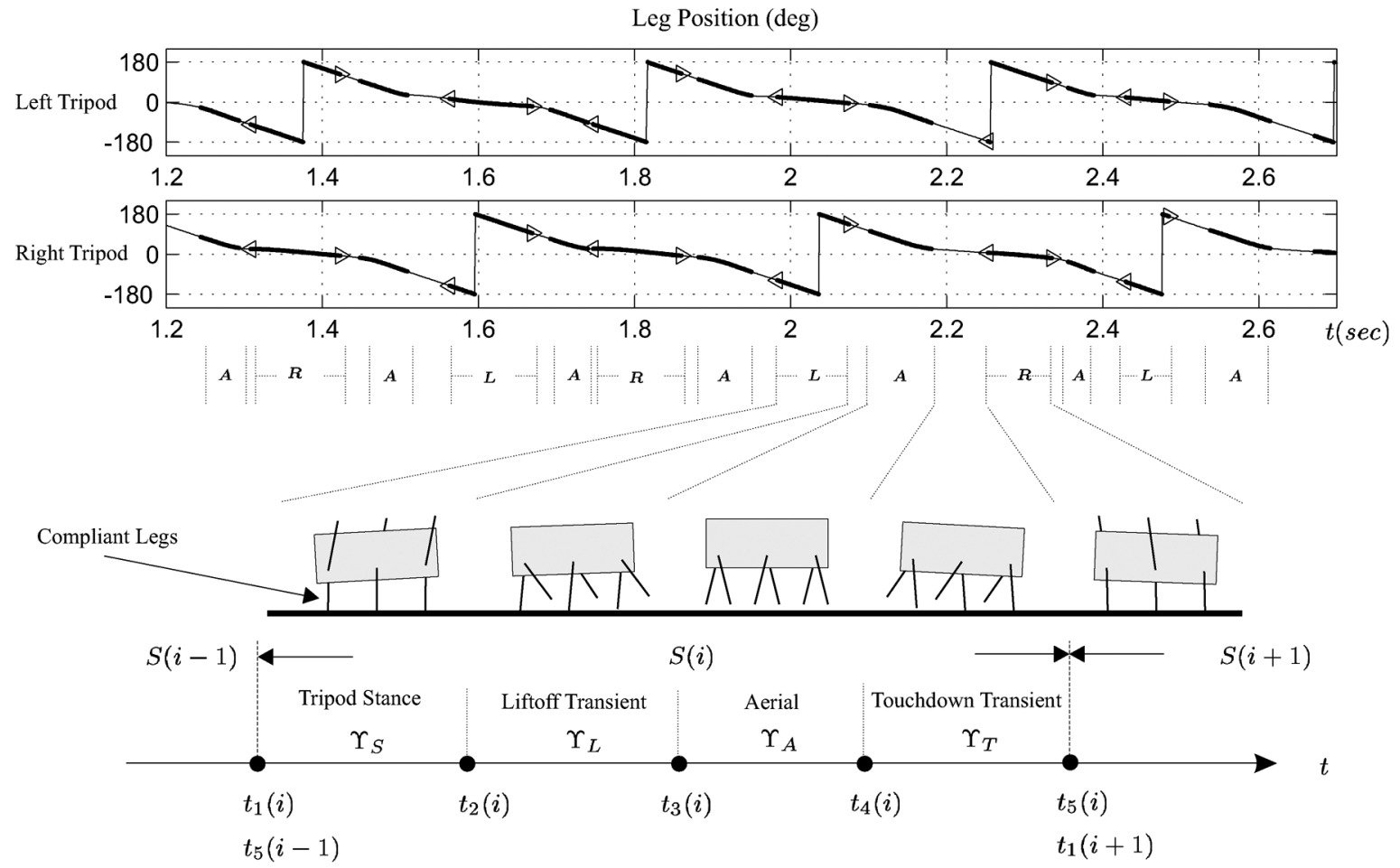

Fig. 2. Conceptual diagram of four consecutive intervals during the $i$ th jogging stride $S(i)$, with data plotted in the top figure illustrating the relation between leg positions $\left(0^{\circ}=\right.$ vertically downward; $\pm 180^{\circ}=$ vertically upward) and four phases (intervals) collected from physical RHex experimental data. Thick line without $\triangleleft \triangleright$ : aerial phase $(|A|)$; thick line with $\triangleleft \triangleright$ : left tripod stance phase $(\vdash L \dashv)$ or right tripod stance phase $(\vdash R \dashv)$; thin line between aerial and tripod stance phases: liftoff/touchdown transient phases $(\|)$.

Nowhere in the databases we have searched ${ }^{4}$ have we come across any paper related to full body state estimation in legged robots by sensor data fusion of IMU with other sensory modalities. Of course, on some level, using a LPS to recalibrate the IMU error is similar to the traditional use of vision/GPS to eliminate drift in the standard mobile robot fusion problem; however, there are important differences between these two "drift-reset" mechanisms. First, for states associated with body localization - center of mass (COM) displacement in the fore/aft and lateral directions together with yaw-the LPS operates at sampling rates as high as those of the IMU, but incurs the threat of drift due to toe slippage [12]. It is best suited for intermediate-range localization (over several strides), as distinct from the long-range localization afforded by relatively infrequent camera/GPS measurements. Second, for states associated with the machine's dynamical stability—pitch, roll, and vertical COM displacement (properties of concern for agile legged robots, but typically not addressed at all in the wheeled robot literature) - the leg sensor provides valuable nondrifting, high-update-rate measurements that are new to the literature (in particular, distinct in their role from those of either differential GPS or vision systems). In summary, we find no prior statement nor solution of the problem posed by this paper: the fusion of leg pose and IMU sensor data for a legged robot with dynamical gaits.

Section II introduces notation and illustrates the nature of the open-loop stabilizing ("jogging") gait [3] we will study.

\footnotetext{
${ }^{4}$ We have searched the Compendex and IEEExplore databases using the key words "legged robot," "state estimation," and "sensor fusion."
}

Section III describes the details of the sensory suite and the acquisition of partial state (body pose from LPS; linear/angular acceleration and angular velocity from AIMU) from raw sensor data. Section IV describes the various dynamical models in each phase of a stride, from which we construct our statistical filters for full body state, along with the details of how to fuse the two independent sensing sources (LPS and IMU). Section V examines the accuracy of the resulting body state estimator implemented offline, on data extracted from numerous runs of the robot RHex pictured in Fig. 1 (bearing the sensor suite described Section III). A brief conclusion follows in Section VI.

\section{DyNAMICAL LOCOMOTION (JOGgING GAIT)}

We defer the fundamental problem of estimator model selection and switching discussed above by recourse to a succession of intuitively appropriate dynamical representations of the "virtual biped" that emerges from well-tuned jogging controllers [3]. We introduce three distinct models in four successively repeating phases-tripod stance phase, liftoff transient phase, aerial phase, and touchdown transient phase-as follows.

Consider the typical sequence of leg contact conditions, depicted in the lower half of Fig. 2, that occurs during steady-state operation in stable dynamical locomotion. During the $i$ th stride interval $S(i):=\left[t_{1}(i) t_{5}(i)\right] \subset R$, a tripod stance interval $\Upsilon_{S}(i):=\left[t_{1}(i) t_{2}(i)\right]$ is succeeded by a period of time when the legs begin to liftoff $\Upsilon_{L}(i):=\left[t_{2}(i) t_{3}(i)\right]$, followed by an interval of aerial flight $\Upsilon_{A}(i):=\left[t_{3}(i) t_{4}(i)\right]$, then touching down through another period of varied leg contacts $\Upsilon_{T}(i):=$ 
$\left[t_{4}(i) t_{5}(i)\right]$ to the fixed tripod stance interval $\Upsilon_{S}(i+1)$ of the next stride $S(i+1)$. We consider the liftoff and touchdown intervals $\Upsilon_{L}(i)$ and $\Upsilon_{T}(i)$ "transients" because they typically exhibit complex sequences of successive leg contacts that reveal little consistent pattern from run to run (or, often, even from stride to stride). In practical implementation, the crucial leg contact information required to detect the onset and termination of each of these phases of a stride may be gleaned directly from the individual leg-strain-based touchdown/configuration sensors [12]. The top half of Fig. 2 shows the relation between these four phases and leg positions of two tripods generated by the custom Buehler Clock [2].

\section{Partial-State Measurements Output DIRECTLY FROM SENSOR}

\section{A. Body Pose From LPS}

A full 6-DOF body pose (COM displacement in lateral $\left(r_{x}\right)$, fore/aft $\left(r_{y}\right)$, vertical $\left(r_{z}\right)$ directions, and body orientation in pitch $(\alpha)$, roll $(\beta)$, and yaw $(\gamma))$ for a hexapod robot in each tripod stance can be obtained from a recently developed novel leg-strain-based configuration measurement system [12]. This body pose estimate is computed using a conventional (memoryless) kinematic model generally expressed in the form

$$
\left[\begin{array}{llllll}
r_{x} & r_{y} & r_{z} & \alpha & \beta & \gamma
\end{array}\right]^{T}=g_{l}\left(\mathbf{s}_{i}, \mathbf{p}_{i}\right)
$$

where $i=1,3,5$ or $i=2,4,6$ denotes left/right tripod, $\mathbf{s}_{i}$ denotes the sensory measurements available regarding the configuration of the kinematic chain connecting the robot body to the $i$ th toe, and $\mathbf{p}_{i}$ denotes the vector from COM to hip of the $i$ th leg. In the case of RHex, with one actuated rotational DOF associated with each compliant leg, $\mathbf{s}_{i}$ can be denoted $\mathbf{s}_{i}=\mathbf{s}_{i}\left(\gamma_{i}, \sigma_{i}\right)=\mathbf{n}_{i}\left(\gamma_{i}\right) \circ \mathbf{m}_{i}\left(\sigma_{i}\right)$, where $\mathbf{n}_{i}$ denotes the $i$ th leg position from encoder measurement, $\gamma_{i}$, and $\mathbf{m}_{i}$ denotes a memoryless transformation from (data-driven phenomenological) models relating leg strain $\sigma_{i}$ to the $i$ th leg configuration, detailed in [12].

\section{B. Linear/Angular Acceleration and Angular Velocity From AIMU With 12-Axis Accelerometer Suite and 3-Axis Gyro}

The TIMU consisting of a 3-axis accelerometer installed at the COM and a 3-axis gyro on the body delivers COM linear acceleration $\left(\ddot{r_{x}}, \ddot{r_{y}}, \ddot{r_{z}}\right)$ and body angular velocity $(\dot{\alpha}, \dot{\beta}, \dot{\gamma})$ in six independent dimensions. In order to obtain angular acceleration data $(\ddot{\alpha}, \ddot{\beta}, \ddot{\gamma})$ as an input to a complete second-order dynamical model for the rotational state, we propose a general method that algebraically computes six independent (translational and rotational) acceleration measurements, as well as three independent angular velocity measurements from a 12 -axis accelerometer suite using the kinematic relationships of rigid body motion, as follows.

The acceleration vector $\mathbf{a}_{p}$ in an inertial "world" frame of a point $p$, rigidly attached to an accelerating "body" frame with origin $o$, is a function of the body's angular velocity $\omega$ and angular acceleration $\dot{\omega}$, as well as the translational acceleration of the origin $\mathbf{a}_{o}$ given by

$$
\mathbf{a}_{p}=\mathbf{a}_{o}+\dot{\omega} \times \mathbf{r}_{o p}+\omega \times\left(\omega \times \mathbf{r}_{o p}\right)
$$

where $\mathbf{r}_{o p}$, the fixed position vector of $p$ relative to the body, is presumed known a priori. In general, we are interested in the motion of the body relative to the world; hence, we seek to extract from measurements of the left-hand quantities information sufficient to derive the right-side unknowns: the COM translational acceleration $\mathbf{a}_{\mathrm{COM}}$ (usually equal to the origin of body frame)

$$
\mathbf{a}_{\mathrm{COM}}=\mathbf{a}_{o}=\left[\begin{array}{lll}
\ddot{r_{x}} & \ddot{r_{y}} & \ddot{r_{z}}
\end{array}\right]^{T}
$$

and the angular acceleration and velocity

$$
\begin{aligned}
\dot{\omega} & =\left[\begin{array}{lll}
\ddot{\alpha} & \ddot{\beta} & \ddot{\gamma}
\end{array}\right]^{T} \\
\omega & =\left[\begin{array}{lll}
\dot{\alpha} & \dot{\beta} & \dot{\gamma}
\end{array}\right]^{T}=\left[\begin{array}{lll}
\omega_{1} & \omega_{2} & \omega_{3}
\end{array}\right]^{T} .
\end{aligned}
$$

Note that the latter three variables appear in a quadratic form; hence, (2) defines a function that is linear in the six unknowns, $\mathrm{a}_{\mathrm{COM}}, \dot{\omega}$, and linear in

$$
q(\omega)=\left[\omega_{i}^{2}+\omega_{j}^{2} \quad \omega_{i} \omega_{j}\right]_{i<j \in 1,2,3}^{T}
$$

the six distinct second-degree monomials of $\omega \times \omega$ formed from the three unknowns of $\omega$. In Appendix I-A, we show how to invert the quadratic map $q$ from $\omega$ to $\omega \times \omega$, thereby establishing that the determination of the nine unknowns in (2) reduces to a linear computation that we will proceed to detail, at the expense of requiring three additional measurements beyond the nine intrinsic dimensions of the data.

The proposed accelerometer suite depicted in Fig. 3 yields a 12-dimensional vector of measurements

$$
\mathbf{a}_{s}:=\left[\begin{array}{llll}
\mathbf{a}_{1}^{T} & \mathbf{a}_{2}^{T} & \mathbf{a}_{3}^{T} & \mathbf{a}_{4}^{T}
\end{array}\right]^{T}
$$

comprised of four distinct spatial acceleration vectors (following upon a very simple calibration procedure from raw accelerometer outputs detailed in Appendix II) $\mathbf{a}_{k}:=\left[\begin{array}{lll}a_{k x} & a_{k y} & a_{k z}\end{array}\right]_{k=1,2,3,4}^{T}$, obtained at the four locations specified by the position vectors

$$
d:=\left[\begin{array}{llll}
d_{1}^{T} & d_{2}^{T} & d_{3}^{T} & d_{4}^{T}
\end{array}\right]^{T} \in R^{12} .
$$

In each case, $k=1,2,3,4$, the measured acceleration vector $\mathbf{a}_{k}$ is linearly related to the 12 unknowns

$$
\mathbf{x}_{\mathrm{var}}=\left[\begin{array}{lll}
\mathbf{a}_{\mathrm{COM}}^{T} & \dot{\omega}^{T} & q(\omega)^{T}
\end{array}\right]^{T}
$$




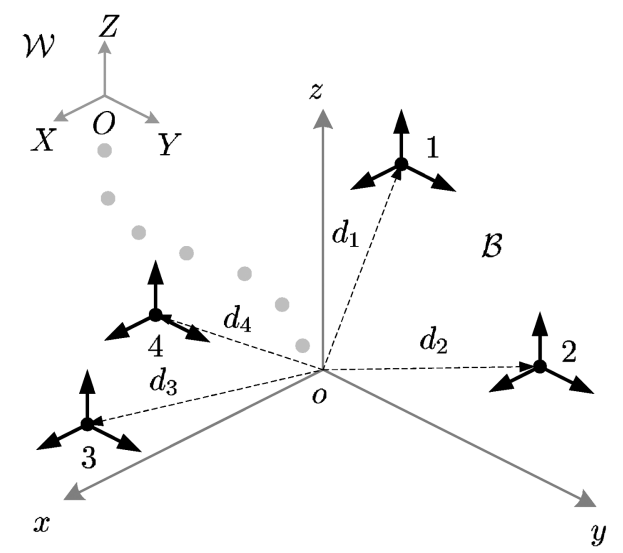

Fig. 3. 12-axis accelerometer suite: measuring 3-axis acceleration in four locations on robot body frame (thick arrows denote the acceleration to be measured).

by a copy of (2), where the role of $\mathbf{r}_{o p}$ is played by $d_{k}$, giving rise to the $12 \times 12$ linear system of equations

$$
\mathbf{a}_{s}=\mathbf{C}(d) \mathbf{x}_{\mathrm{var}} .
$$

Since $d$ is known a priori, the extraction of the desired acceleration and angular velocity data $\mathbf{x}_{\mathrm{var}}$ now hinges upon the rank and numerical condition of the "structure" matrix $\mathbf{C}(d)$.

We observe that the determinant of the $12 \times 12$ matrix $\mathbf{C}(d)$, $\operatorname{det}(\mathbf{C}(d))$, is given by the determinant of the "sensor simplex" array, $\operatorname{det}(D)$

$$
\operatorname{det}(\mathbf{C}(d))=(2 \operatorname{det}(D))^{3}
$$

where $D:=\left[\begin{array}{lll}d_{2}-d_{1} & d_{3}-d_{1} & d_{4}-d_{1}\end{array}\right]$. Hence, so long as the accelerometer suite of Fig. 3 defines a spatial tetrahedron with nonzero volume (that is, the four accelerometers are in a general position, such that there is no coplanar subset of any three of them), it provides, in principle, a complete AIMU: a means of extracting full 6-DOF rigid-body acceleration and 3-DOF angular velocity data, with no recourse to rate gyros at all.

Although the determinant of the structure matrix $\mathbf{C}(d)$ can be shown to reduce to that of the "simplex matrix" $D$, its condition number (i.e., the ratio of the largest to the smallest eigenvalues of the symmetric square $\mathbf{C}^{T} \mathbf{C}$ ) is a more complicated function of the shape of the tetrahedron (or, equivalently, the frame of reference) the simplex matrix defines. Not surprisingly, the condition number is invariant to rotations of this tetrahedron around the COM; hence, the optimal condition occurs when it is placed symmetrically coincident with the corners of a cube. However, because the structure matrix combines entries with and without physical scale (length $/ s^{2}$ and $1 / s^{2}$ both in the same rows), it turns out there is actually a preferred linear dimension of this cube at which the resulting condition number is optimal. Moreover, since the singular values (functions of the eigenvalues of the of symmetric square $\mathbf{C}^{T} \mathbf{C}$ ) are determined by high-order polynomials (in the entries of the tetrahedron $D$ ), there is every reason to expect that the condition would be very sensitive to the "shape" and "size" of the tetrahedron and its location relative to COM.
Indeed, in practice, numerical exploration suggests that unit dimensions whose associated cubes lie completely within the robot's body cannot be sufficiently accurately resolved spatially by simple physical measurement to derive adequate advantage from the improved condition of the resulting structure matrix. In particular, as we detail in Appendix I-B, the largest volume tetrahedron inscribed within RHex's rectangular body yields a very stiff structure matrix $\mathbf{C}(d)$, whose large singular values are associated with the translational and rotational acceleration components of $\mathbf{x}_{\mathrm{var}}$, and whose (uselessly small) singular values are associated with the rotational velocity components $q(\omega)$ of $\mathbf{x}_{\mathrm{var}}$. Consequently, we rely upon measurements of $\omega$ arising from a MEMS gyro, retaining only the acceleration components $\left(\mathbf{a}_{\mathrm{COM}}\right.$ and $\left.\dot{\omega}\right)$ of the accelerometer suite's estimate for $\mathbf{x}_{\mathrm{var}}=$ $\mathbf{C}^{-1}(d) \mathbf{a}_{s}$, namely

$$
\begin{aligned}
\mathbf{a}_{\mathrm{COM}} & =g_{a}\left(\mathbf{a}_{k}, d_{k}\right)_{k=1,2,3,4} \\
\dot{\omega} & =g_{r}\left(\mathbf{a}_{k}, d_{k}\right)_{k=1,2,3,4} .
\end{aligned}
$$

\section{Fusion Algorithms For Full Body State Estimation}

Tradeoffs between the performance (accuracy, reliability) and cost (actual dollars, required "real estate," and ease of use) of a sensor suite are governed by tightly interrelated issues arising from geometric, as well as technological, considerations. Once the sensor is chosen, however, the only possibility for improved state estimates depends on the choice of estimation model and algorithm. The design of estimation algorithms for nonlinear dynamical systems has spawned a huge body of literature whose consideration lies well beyond the scope of this paper. Hence, we concentrate our efforts on a well-understood and highly regarded standard, the EKF, and devote the remainder of the paper to a comparison of estimates arising from different dynamical models that make varying use of the available sensor suite.

As discussed in Section II, we adopt a greatly simplified view of hexapod jogging by positing a succession of low-dimensional models presumed to capture the essential features of the robot's rigid body dynamics, as determined by an idealized periodic sequence of leg contact conditions. We trigger the succession of one model by the next using a "hard switch," a deterministic predicate over the raw sensory data, and initialize the successor using an exact copy of the predecessor's final state. In future work, we will take a more formal point of view, and seek to implement theoretically motivated switching procedures based upon a comparison of the multiple models' prediction errors, against which the results of this preliminary inquiry may be compared.

We are also interested in assessing the relative value of the two sensing modalities, and will adjust the details of the successive estimation models to accommodate the presence or absence of appropriate subsets of the complete sensorium. We find it most natural to treat the LPS system, operative only during intermittent stance phases, as a "drift corrector" for the IMU sensors that runs continually through aerial, as well as ground contact, phases. We seek to determine whether one of these two subsystems is "better" than the other, and whether two operating together in this manner are better than either one alone.

In this section, we first briefly review the EKF structure as a means of establishing notational conventions. Models 
and resulting filters for the rotational DOFs are presented in Section IV-B, and those addressing the translational DOFs in Section IV-C. Finally, Section IV-D presents our methodology to compute full body state from each of the two independent sensing sources alone.

\section{A. Notation Associated With the EKF}

Given a discrete time-invariant plant $\mathbf{x}_{k+1}=f\left(\mathbf{x}_{k}, \mathbf{u}_{k}, \mathbf{w}_{k}\right)$ with measurement $\mathbf{y}_{k+1}=h\left(\mathbf{x}_{k}, \mathbf{v}_{k}\right)$, where the process noise $\mathbf{w}_{k}$ and measurement noise $\mathbf{v}_{k}$ are white with zero means and covariance defined by $\mathcal{E}\left[\mathbf{w}_{k} \mathbf{w}_{k}^{T}\right]=\mathbf{Q}_{k}$ and $\mathcal{E}\left[\mathbf{v}_{k} \mathbf{v}_{k}^{T}\right]=\mathbf{R}_{k}$, an EKF incorporates two steps: a time update (a priori estimate)

$$
\begin{aligned}
\mathbf{x}_{k+1}^{-}= & f\left(\mathbf{x}_{k}^{-}, \mathbf{u}_{k}, 0\right) \\
\mathbf{P}_{k+1}^{-}= & \Phi_{k} \mathbf{P}_{k}^{+} \Phi_{k}^{T}+\mathbf{Q}_{k} \\
& \text { with } \Phi_{k}=\left.\frac{\partial f}{\partial x}\right|_{\mathbf{x}_{k}^{+}} \text {and } \Gamma_{k}=\left.\frac{\partial f}{\partial x}\right|_{\mathbf{u}_{k}}
\end{aligned}
$$

and a measurement update (a posteriori estimate)

$$
\begin{aligned}
\mathbf{K}_{k+1}= & \mathbf{P}_{k+1}^{-} \mathbf{H}_{k}^{T}\left(\mathbf{H}_{k} \mathbf{P}_{k+1}^{-} \mathbf{H}_{k}^{T}+\mathbf{R}_{k}\right)^{-1} \\
\mathbf{x}_{k+1}^{+}= & \mathbf{x}_{k+1}^{-}+\mathbf{K}_{k+1}\left(\mathbf{z}_{k}-\mathbf{H}_{k} \mathbf{x}_{k+1}^{-}\right) \\
\mathbf{P}_{k+1}^{+}= & \mathbf{P}_{k+1}^{-}-\mathbf{K}_{k+1} \mathbf{H}_{k} \mathbf{P}_{k+1}^{-} \\
& \text {with } \mathbf{H}_{k}=\left.\frac{\partial h}{\partial x}\right|_{\mathbf{x}_{k}^{+}}
\end{aligned}
$$

where $\mathbf{P}_{k}$ is the error covariance matrix, $\mathbf{K}_{k}$ is the so-called Kalman gain, and $\mathbf{z}_{k}$ is the sensor measurement (partial state measured directly from sensors of the kind discussed in Section III, and to be detailed in the succeeding two subsections). Upon initializing the value of state $\mathbf{x}_{0}$ and error covariance matrix $\mathbf{P}_{0}$, the EKF continuously delivers the "optimal" state estimates by consecutively performing these two updates at each time stamp.

The body pose measurement noise covariance arising from the LPS $\left(\mathbf{R}_{l}\right)$, along with the linear/angular acceleration noise covariance arising from the 12 -axis accelerometer suite $\left(\mathbf{R}_{a} / \mathbf{R}_{r}\right)$ are propagated at nominal points ( $\mathbf{s}_{0}$ and $\mathbf{a}_{0}$ ) of raw sensor noise $\left(\mathbf{R}_{\mathbf{s}_{i}}\right.$ and $\left.\mathbf{R}_{\mathbf{a}_{k}}\right)$ from empirical measurement

$$
\begin{array}{ll}
\mathbf{R}_{l}=\mathbf{J}_{l} \mathbf{R}_{\mathbf{s}_{i}} \mathbf{J}_{l}^{T} \quad \text { with } \mathbf{J}_{l}=\left.\frac{\partial g_{l}}{\partial \mathbf{s}_{i}}\right|_{\mathbf{s}_{0}} \quad i=1,3,5 \text { or } i=2,4,6 \\
\mathbf{R}_{a}=\mathbf{J}_{a} \mathbf{R}_{\mathbf{a}_{k}} \mathbf{J}_{a}^{T} \quad \text { with } \mathbf{J}_{a}=\left.\frac{\partial g_{a}}{\partial \mathbf{a}_{k}}\right|_{\mathbf{a}_{0}} \quad k=1,2,3,4 \\
\mathbf{R}_{r}=\mathbf{J}_{r} \mathbf{R}_{\mathbf{a}_{k}} \mathbf{J}_{r}^{T} \quad \text { with } \mathbf{J}_{r}=\left.\frac{\partial g_{r}}{\partial \mathbf{a}_{k}}\right|_{\mathbf{a}_{0}} \quad k=1,2,3,4
\end{array}
$$

where $g_{l}$ is defined in (1), and $g_{a}$ and $g_{r}$ are defined in (4). The noise covariance of angular velocity from gyro $\left(\mathbf{R}_{g}\right)$ is directly measured from sensor noise empirically.

\section{B. A Posteriori Models for Estimation of Rotational State}

We find it convenient to adopt the quaternion representation of rigid body rotations, $q \in S^{3}$ (i.e., unit vectors in $R^{4}$ ), in which case velocities are tangent vectors to the sphere $\omega \in R^{3}$, yielding the complete rotational state representation as

$$
\mathbf{x}_{7}=\left[\begin{array}{lllllll}
q_{0} & q_{1} & q_{2} & q_{3} & \omega_{1} & \omega_{2} & \omega_{3}
\end{array}\right]^{T} .
$$

When we introduce angular acceleration inputs from the 12-axis accelerometer suite to this model, we require an appropriately "inflated" view of state

$$
\mathbf{x}_{10}=\left[\begin{array}{llllllllll}
q_{0} & q_{1} & q_{2} & q_{3} & \omega_{1} & \omega_{2} & \omega_{3} & \dot{\omega}_{1} & \dot{\omega}_{2} & \dot{\omega}_{3}
\end{array}\right]^{T} .
$$

For purposes of sensor fusion in all phases of the recurring gait cycle, our choice of a priori model (5) follows the long tradition in the inertial guidance literature [15] of constant acceleration, which simply asserts that the position is the integral of velocity, which is, in turn, the integral of acceleration. Differing sensory feedback structures yield different a posteriori estimates through the substitution of differing output maps $h$ and $\mathbf{H}$, as follows.

1) Fusion of LPS and AIMU: For the aerial and transient phases with only the AIMU (angular velocity and acceleration) available, sensor measurement vector $(\mathbf{z})$ and measurement matrix $(\mathbf{H})$ shown in (6) are

$$
\begin{aligned}
\mathbf{z}_{\mathrm{AIMU}} & =\left[\begin{array}{llllll}
\omega_{1} & \omega_{2} & \omega_{3} & \dot{\omega}_{1} & \dot{\omega}_{2} & \dot{\omega}_{3}
\end{array}\right]^{T} \\
\mathbf{H}_{\mathrm{AIMU}} & =\left[\begin{array}{lll}
\mathbf{O}_{6 \times 4} & \mathbf{I}_{6 \times 6}
\end{array}\right]
\end{aligned}
$$

where $\mathbf{O}_{m \times n}$ and $\mathbf{I}_{m \times n}$ are the zero and identity matrices with dimension $m \times n$. In tripod stance phase with data from the LPS as well, sensor measurement available in all 10-element state yields $\mathbf{z}$ being exactly the same as the state vector shown in (8), which results in $\mathbf{H}$ being identity

$$
\begin{aligned}
\mathbf{z}_{\text {AIMU }+ \text { LPS }} & =\mathbf{x}_{10} \\
\mathbf{H}_{\text {AIMU }+ \text { LPS }} & =\mathbf{I}_{10 \times 10} .
\end{aligned}
$$

The addition of LPS data in the tripod stance phase renders this version of the EKF observable; the formal interpretation of its intuitive "drift-killing" effect relative to the nominal (formally unobservable) version of the EKF endowed with only IMU data in the prior aerial and transient phases.

2) Fusion of LPS and TIMU: We also fuse the LPS data with the TIMU in order to evaluate the effect of angular acceleration by comparing the performance of this system with the previous system with extra angular acceleration input in AIMU. In this case, $\mathbf{x}_{7}$, in (7), is adopted as the state representation. For the aerial and transient phases with only the TIMU (angular velocity) available, sensor measurement vector $(\mathbf{z})$ and measurement matrix $(\mathbf{H})$ shown in (6) are

$$
\begin{aligned}
\mathbf{z}_{\text {TIMU }} & =\left[\begin{array}{lll}
\omega_{x} & \omega_{y} & \omega_{z}
\end{array}\right]^{T} \\
\mathbf{H}_{\text {TIMU }} & =\left[\begin{array}{lll}
\mathbf{O}_{3 \times 4} & \mathbf{I}_{3 \times 3}
\end{array}\right] .
\end{aligned}
$$

In tripod stance phase with data from the LPS, as well, $\mathbf{z}$ and $\mathbf{H}$ yield

$$
\begin{aligned}
\mathbf{z}_{\mathrm{TIMU}+\mathrm{LPS}} & =\left[\begin{array}{lllllll}
q_{0} & q_{1} & q_{2} & q_{3} & \omega_{x} & \omega_{y} & \omega_{z}
\end{array}\right]^{T} \\
\mathbf{H}_{\mathrm{TIMU}+\mathrm{LPS}} & =\mathbf{I}_{7 \times 7}
\end{aligned}
$$

where $\left[\begin{array}{llll}q_{0} & q_{1} & q_{2} & q_{3}\end{array}\right]^{T}=f_{q}(\alpha, \beta, \gamma)$ is the quaternion representation of orientation obtained from the LPS. 


\section{A Posteriori Models for Estimation of Translational State}

Proceeding with the same naive assumption of constant acceleration, translational motion along three principal axes can be modeled independently. In contrast to the rotational DOFs, there is no intrinsic sensory measure of translational velocity, and the use of body acceleration data must be mediated by the rotational estimates relative to the inertial frame with appropriate gravity compensation.

For each translational DOF, we require a 3-D state including acceleration given by

$$
\mathbf{x}_{3}=\left[\begin{array}{lll}
r & \dot{r} & \ddot{r}
\end{array}\right]^{T}
$$

due to the fact that acceleration is the principal measurement. The translational state only has two kinds of sensor feedback: acceleration from IMU and body pose from LPS (for translational state AIMU is equal to TIMU). Similar to the rotational case, in aerial and transient phases with only IMU available, yields $\mathbf{H}$ as

$$
\mathbf{H}_{\mathrm{IMU}}=\left[\begin{array}{lll}
0 & 0 & 1
\end{array}\right] \text {. }
$$

In tripod stance phase with data from LPS as well, $\mathbf{H}$ becomes

$$
\mathbf{H}_{\mathrm{IMU}+\mathrm{LPS}}=\left[\begin{array}{lll}
1 & 0 & 0 \\
0 & 0 & 1
\end{array}\right]
$$

\section{A Priori and A Posteriori Models for Estimation of Full Body State From Each Sensor Alone}

We finally detail the nature of the models (5) and (6) used to form full body state estimates for the LPS and IMU sensors, each in isolation.

1) Body State From LPS: During the tripod stance phase $\left(\Upsilon_{S}(i)\right)$, the LPS delivers 6-DOF body pose data with respect to the initial touchdown frame of reference. The stance pose and velocity (formed by differentiating recent pose data) state estimates at the moment of takeoff are handed off as the initial condition for the subsequent transient phase. In this transient phase, state estimates arise from a constant velocity model, which replaces the constant acceleration model of the previous phase to avoid noisy initial conditions associated with doubly differentiated takeoff acceleration data. A ballistic flight model of the succeeding flight phase is again initialized by the state of the estimator at the conclusion of the transient phase. Another transient phase, structured in the same way as the first, then precedes a touchdown event that initiates the next stance phase. These estimates, based on carrying forward the most recent stance phase data via abstract kinematic models, assuming the absence of any physical measurements during transient and aerial phases, can now be compared with those obtained using an EKF to process supplemental IMU data.

2) Body State From TIMU: Full body state from TIMU can be directly obtained by double/single integration of raw accelerometer/gyro data, or by using the Kalman filter (KF)/EKF described in Section IV-A, based upon the naive constant acceleration model already discussed in Section IV-B and C. The a posteriori model (6) using a sensor measurement projected appropriately from the components in (7) is exactly the same as (9). It has long been remarked in the literature that since its dynamical model is unobservable, the associated KF of this "IMU only" system does not guarantee better performance than direct integration. Of course, the naive assumption of white noise and likely inaccurate initial error covariance matrix add to the accumulation of error.

\section{EXPERIMENT RESULTS}

\section{A. Experiment Setup}

We have evaluated these estimators using offline data gathered during numerous physical experimental jogging runs with a version of RHex $(25 \mathrm{~cm} \times 50 \mathrm{~cm} \times 30 \mathrm{~cm})$ pictured in Fig. 1, incorporating the required sensors, which include the customized LPS (delivering full 6-DOF body pose) detailed in [12], a 3-axis rate gyro (by three 1-axis MEMS gyro ADXRS300 from Analog Device, delivering angular velocity), and a 12-axis accelerometer suite (by eight 2-axis MEMS accelerometer ADXL210 from Analog Device, delivering linear/angular acceleration). All three kinds of sensors, leg pose, gyro, and accelerometer, have turn-on bias calibration setup implemented in the software. In principle, as we have discussed in Section III-B and detailed in Appendix I, the 12-axis accelerometer suite can play the role of a complete AIMU, delivering three components of body state (linear and angular acceleration, as well as angular velocity) without the need for a gyro as long as the "sensor simplex" satisfies the volume property introduced in Section III-B. While RHex's dimensions permit a sensor arrangement that formally satisfies this requirement, we detail in Appendix I-B how the robot's relatively small body constrains the numerical conditioning of the resulting regression problem severely enough to preclude the use of angular velocity estimates so derived. Consequently, we find it essential to use the rate gyro data included in the IMU package.

To assess performance improvements resulting from the fusion of leg pose and IMU data, we have run RHex under the Ground Truth Measurement System (GTMS), the independent visual GTMS detailed in [12] and [24]. This yields another set of 12-DOF body state (6 DOFs from position measurement and 6 DOFs from their derivatives) for comparison. We quantify performance by presenting the standard root mean squared (rms) error given by

$$
\chi(l, \hat{l}):=\sqrt{\left(\|l-\hat{l}\|_{2}^{2} / M\right)}
$$

where $l$ represents the state from GTMS, $\hat{l}$ denotes the same state from output of the algorithm, and $M$ is the length of the data.

The common sensor data for all models and associated GTMS output is recorded over the course of 2-m-long experimental runs and then processed offline to assess model performance. We observed neither saturation nor even noticeable changes in 
TABLE I

EMPIRICAL PHASE RELATIONS IN RHEX JOGGING GAIT

\begin{tabular}{|l|c|c|}
\hline \multicolumn{3}{|c|}{ Phases in one complete stride } \\
\hline Phases & $\begin{array}{c}\text { Average time (ms) } \\
\text { mean (std) }\end{array}$ & $\begin{array}{c}\text { Percentage of time (\%) } \\
\text { mean (std) }\end{array}$ \\
\hline Tripod Stance & $96.1(5.9)$ & $44.4(2.7)$ \\
\hline Liftoff Transient & $29.0(4.9)$ & $13.4(2.3)$ \\
\hline Aerial & $55.2(6.6)$ & $25.5(3.0)$ \\
\hline Touchdown Transient & $36.0(5.5)$ & $16.7(2.6)$ \\
\hline \hline Total & $216.3(2.3)$ & 100.0 \\
\hline
\end{tabular}

acceleration magnitude due to leg impacts (at our 1-kHz sampling rate), which we attribute to energy absorption by the compliant legs and rubber feet. RHex's relatively constrained kinematics precludes the exercise of its yaw DOF, barring intentional excitation of slipping motion on particular toes, such as would be required for turning. In consequence, we perform straightline experimental runs on flat terrain, because turns are difficult to execute repeatably under the current open-loop gait controller. This simplified evaluation protocol avoids the need for another (complex and necessarily empirical) model that describes turning. Similarly, since we are only concerned with stable gaits in this paper, we analyze data gathered only during steady-state conditions, following the transient from standstill to exclude irregular model switching sequences and the attendant model errors that would not be captured well within the EKF's Gaussian noise framework. Table I summarizes RHex's absolute and relative phase timing measured at $1 \mathrm{KHz}$ averaged over 10 experimental runs, providing a feel for how quickly the phases switch and how many data points are available in each phase. Individual leg strain measurements are used to estimate the constituent leg touchdown and liftoff times. The small variation of total time in one complete stride demonstrates the overall reliability of the steady-state stride excited by the Buehler clock [2], whereas the larger relative variations of each phase suggest the imperfections in the gait stability that result from this feedforward control scheme.

\section{B. Performance Evaluation of Rotational State}

Table II presents the rms difference (mean and standard deviation from 10 experimental runs) between the GTMS output and each of four alternative algorithms (filtering only the LPS data, only the TIMU, or fusion of data from both the LPS and TIMU or AIMU) for all six rotational states, including body orientation in pitch $(\alpha)$, roll $(\beta)$, yaw $(\gamma)$, and their derivatives $(\dot{\alpha}, \dot{\beta}, \dot{\gamma})$. The LPS alone offers good orientation estimation in the tripod stance phase [12]. However, its performance in overall jogging locomotion is poor due to the absence of output throughout the remaining three phases, where state estimates are simply predictions from a very imperfect model applied to those estimated at takeoff. This distinction is evident in Fig. 4, a plot of the four traces (those of the GTMS and the three algorithms) over one stride of one of the runs. The LPS-based estimates match well the GTMS data during stance $(|T|)$, but do fail to track in the remaining phases. In contrast, the TIMU alone exhibits good angular velocity estimates, but suffers the expected drift in angular position accuracy in consequence of blind integration. These complementary strengths and weaknesses are nicely exploited by the fused algorithm in which the LPS acts as a "recalibration" mechanism during the tripod stance phase to kill the drift in the IMU computation, yielding the significant improvement of the orientation estimates. Moreover, with the contribution of additional angular acceleration data, ${ }^{5}$ fusion system further improves the performance of velocity estimates. ${ }^{6}$

\section{Performance Evaluation of Translational State}

Table III presents the rms difference (mean and standard deviation from 10 experimental runs) between the GTMS output and the same three alternative algorithms (filtering only the LPS data, only the TIMU, and fusion of the LPS and IMU measurements) ${ }^{7}$ for all 6-DOF translational body states, including COM displacement in lateral $\left(r_{x}\right)$, fore/aft $\left(r_{y}\right)$, vertical $\left(r_{z}\right)$ directions, and their derivatives $\left(\dot{r}_{x}, \dot{r}_{y}, \dot{r}_{z}\right)$. Fig. 5 plots COM displacement estimates produced by each algorithm and GTMS (dot) for one of the typical experimental runs. Estimates produced by filtering only the LPS data (dash-dotted line) exhibit performance that is good in displacement, but poor in velocity, due to the noise-amplifying differentiation it entails. Though performance of velocity estimation by IMU only in Table III seems to be similar to that by LPS in consequence of integration error, Fig. 5 does reveal good tracking ability in the velocity state from IMU (slope or high-frequency components in plot, especially noticeable in $r_{x}$ in Fig. 5). This further suggests that with proper "recalibration" to kill the integration error, the IMU will deliver better velocity estimation. Thus, the dramatically different strengths of these two sensors provide the underlying motivation for fusing sensor data in the first place, with the anticipated improvements in performance. ${ }^{8}$

\section{CONCLUSION}

We have developed a hybrid full 12-DOF body state estimator for a hexapod robot executing a steady jogging gait with a significant aerial phase. The estimator is presently implemented offline on data collected by a low-cost MEMS-based IMU and a novel leg-strain-based body pose estimator installed on a copy of the robot RHex [2]. The proper fusion of these data yield state estimates that agree with measurements taken by an offboard GTMS (up to its noise floor), whereas, in contrast, neither sensing modality yields comparable accuracy when used in isolation. The associated computational costs are not excessive, and the algorithm is now ready for online implementation on RHex in conjunction with more aggressive state-based feedback controllers [11], [26]. The sensor suite is quite generic, and we

\footnotetext{
${ }^{5}$ More detailed comparisons of results formed using angular acceleration data from the AIMU against results using angular velocity from gyro only can be found in [25].

${ }^{6}$ Although there is no algorithmic obstacle to fusing yaw data, RHex kinematics precludes yaw output from the LPS, so the fused filter actually produces yaw and yaw velocity estimates directly from the AIMU.

${ }^{7}$ Recall that the AIMU differs from the TIMU by incorporating extra angular acceleration data, whereas for the translational state, these two systems are the same.

${ }^{8}$ Instead of the constant acceleration model, in the aerial phase, we also evaluate the performance of a physical-based ballistic model which, expectably, results in similar performance. This further confirms correct calibration and gravity compensation of accelerometers, since measurement of acceleration should show "ballistic flight" in the aerial phase [25].
} 
TABLE II

RMS ERror of Rotational Body State Estimation ACCording to LPS Alone, TIMU Alone, AND Fusion Algorithm (LPS Plus TIMU OR AIMU) DETAILED IN SECTION IV-D AND SECTION IV-B

\begin{tabular}{|c|c|c|c|c|c|c|c|}
\hline & Model Type & \multicolumn{3}{|c|}{ Body State - Angular Velocity } & \multicolumn{3}{|c|}{ Body State - Orientation } \\
\hline & & $\begin{array}{c}\dot{\alpha} \\
(\mathrm{deg} / \mathrm{s}) \\
\text { mean }(\mathrm{std})\end{array}$ & $\begin{array}{c}\dot{\beta} \\
(\mathrm{deg} / \mathrm{s}) \\
\text { mean (std) }\end{array}$ & $\begin{array}{c}\dot{\gamma} \\
(\mathrm{deg} / \mathrm{s}) \\
\text { mean (std) }\end{array}$ & $\begin{array}{c}\alpha \\
(\mathrm{deg}) \\
\text { mean (std) }\end{array}$ & $\begin{array}{c}\beta \\
(\mathrm{deg}) \\
\text { mean (std) }\end{array}$ & $\begin{array}{c}\gamma \\
\text { (deg) } \\
\text { mean (std) }\end{array}$ \\
\hline a & Leg Pose Sensor & $43.41(6.47)$ & $74.34(6.35)$ & N/A & $1.65(0.29)$ & $5.30(0.58)$ & N/A \\
\hline $\mathrm{b}$ & Traditional IMU (with EKF) & $16.21(4.06)$ & $41.24(7.75)$ & $9.07(1.66)$ & $1.42(0.79)$ & $2.38(0.79)$ & $1.09(0.56)$ \\
\hline $\mathrm{C}$ & Fusion (Leg Pose Sensor and Traditional IMU) & $16.52(4.40)$ & $41.24(7.78)$ & $9.07(1.66)$ & $0.96(0.21)$ & $1.71(0.49)$ & $1.09(0.56)$ \\
\hline $\mathrm{D}$ & Fusion (Leg Pose Sensor and Advanced IMU) & $15.32(3.84)$ & $37.07(7.52)$ & $8.43(1.53)$ & $0.96(0.20)$ & $1.75(0.48)$ & $1.19(0.60)$ \\
\hline
\end{tabular}
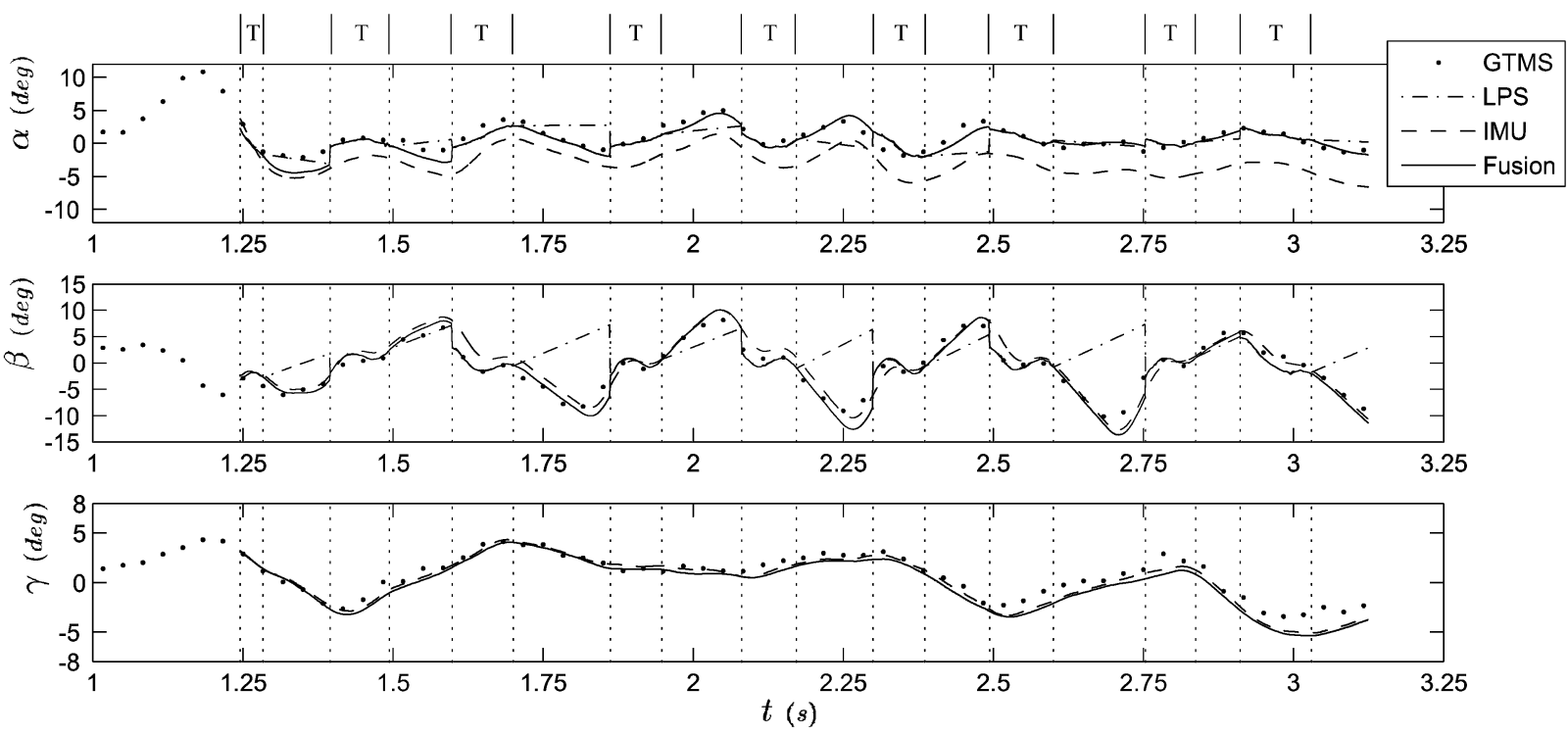

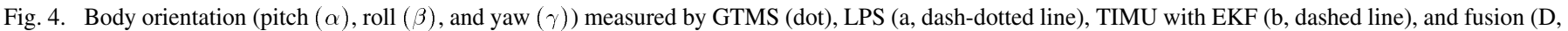

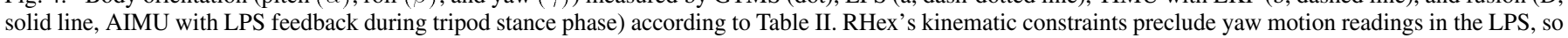

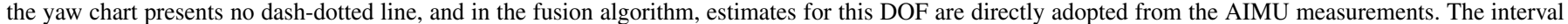
$|T|$ denotes tripod stance phase as determined by the leg-strain-based touchdown/configuration sensor [12]

are proceeding with an implementation of this algorithm upon other legged running machines, as well.

Our results corroborate the notion that two distinct sensory modalities with complementary strengths and weaknesses should yield better state estimates in combination than either can deliver alone. The LPS cannot function in flight, but delivers reliable position information during stance, when the legs do not slip. However, velocity data can only be derived from numerical differentiation, which is bound to inject noise. In contrast, the IMU operates continually through flight and stance, and offers reliable velocity information, so long as the gyros do not saturate. However, position data can only be derived from numerical integration, and an increasing drift arising from integration error is likely. For both translational and rotational states, performance is significantly increased when both sensor modalities are fused appropriately.

Using the "grounded" LPS to complement drifting IMU-based estimates of pitch and roll, and to correct COM vertical displacement estimates, provides a "recalibration" in stance that insures bounded estimation errors in the final outputs. IMU integration drift in the remaining state components can also be mitigated over short distances by this leg-based "recalibration," but slipping toes will eventually degrade absolute horizontal displacement estimates, in analogy to the well-known problems of odometry in wheeled vehicles [12]. To kill drift over longer ranges, we would require such modalities as a GPS (for COM lateral and fore/aft motion) with a magnetometer (for yaw motion) or a vision system for absolute localization.

Endowed with the fusion algorithm we have described, we believe that the combined LPS and AIMU provide a ready platform for "slow" (i.e., stride-to-stride level) real-time feedback control of steady-state gait parameters, and our near-term plans are to begin experimental work of this kind. Before these sensors can provide state estimates suitable for "fast" (within stride) control of badly perturbed transient body states, there are two additional improvements that seem essential. First, we will need to develop more physically realistic dynamical models of the transient states, a simple example being the spring-loaded inverted pendulum model for the tripod stance phase [27]. We are convinced there is a need for still more complex combinations of physically motivated ground-contact models during the touchdown and liftoff transient phases to improve state estimation and reduce transience in each phase. Second, we require further algorithmic means of triggering the hybrid dynamics switching protocols that were effected in this paper only by reference to 
TABLE III

RMS ERror of Translational Body State Estimation AcCording to LPS Alone, IMU Alone, AND Fusion ALGORITHM DETAILED IN SECTION IV-D AND SECTION IV-C

\begin{tabular}{|c|l|c|c|c|c|c|c|}
\hline & Model Type & \multicolumn{3}{|c|}{ Body State - COM Velocity } & \multicolumn{3}{|c|}{ Body State - COM displacement } \\
\hline & & $\begin{array}{c}\dot{r}_{x} \\
(\mathrm{~cm} / \mathrm{s}) \\
\text { mean }(\mathrm{std})\end{array}$ & $\begin{array}{c}\dot{r}_{y} \\
(\mathrm{~cm} / \mathrm{s}) \\
\text { mean }(\mathrm{std})\end{array}$ & $\begin{array}{c}\dot{r}_{z} \\
(\mathrm{~cm} / \mathrm{s}) \\
\text { mean }(\mathrm{std})\end{array}$ & $\begin{array}{c}r_{x} \\
(\mathrm{~cm}) \\
\text { mean }(\mathrm{std})\end{array}$ & $\begin{array}{c}r_{y} \\
(\mathrm{~cm}) \\
\text { mean }(\mathrm{std})\end{array}$ & $\begin{array}{c}r_{z} \\
(\mathrm{~cm}) \\
\mathrm{mean}(\mathrm{std})\end{array}$ \\
\hline $\mathrm{a}$ & Leg Pose Sensor & $21.84(1.75)$ & $17.39(4.30)$ & $14.88(1.63)$ & $4.16(3.52)$ & $5.42(3.36)$ & $0.82(0.14)$ \\
\hline $\mathrm{b}$ & IMU (with KF) & $32.29(21.10)$ & $20.46(15.32)$ & $10.60(3.30)$ & $23.60(14.80)$ & $16.14(14.18)$ & $5.25(4.00)$ \\
\hline D & Fusion (Leg Pose Sensor and IMU) & $10.48(3.74)$ & $7.41(2.41)$ & $9.83(3.23)$ & $4.74(3.17)$ & $5.85(3.74)$ & $1.10(0.41)$ \\
\hline
\end{tabular}
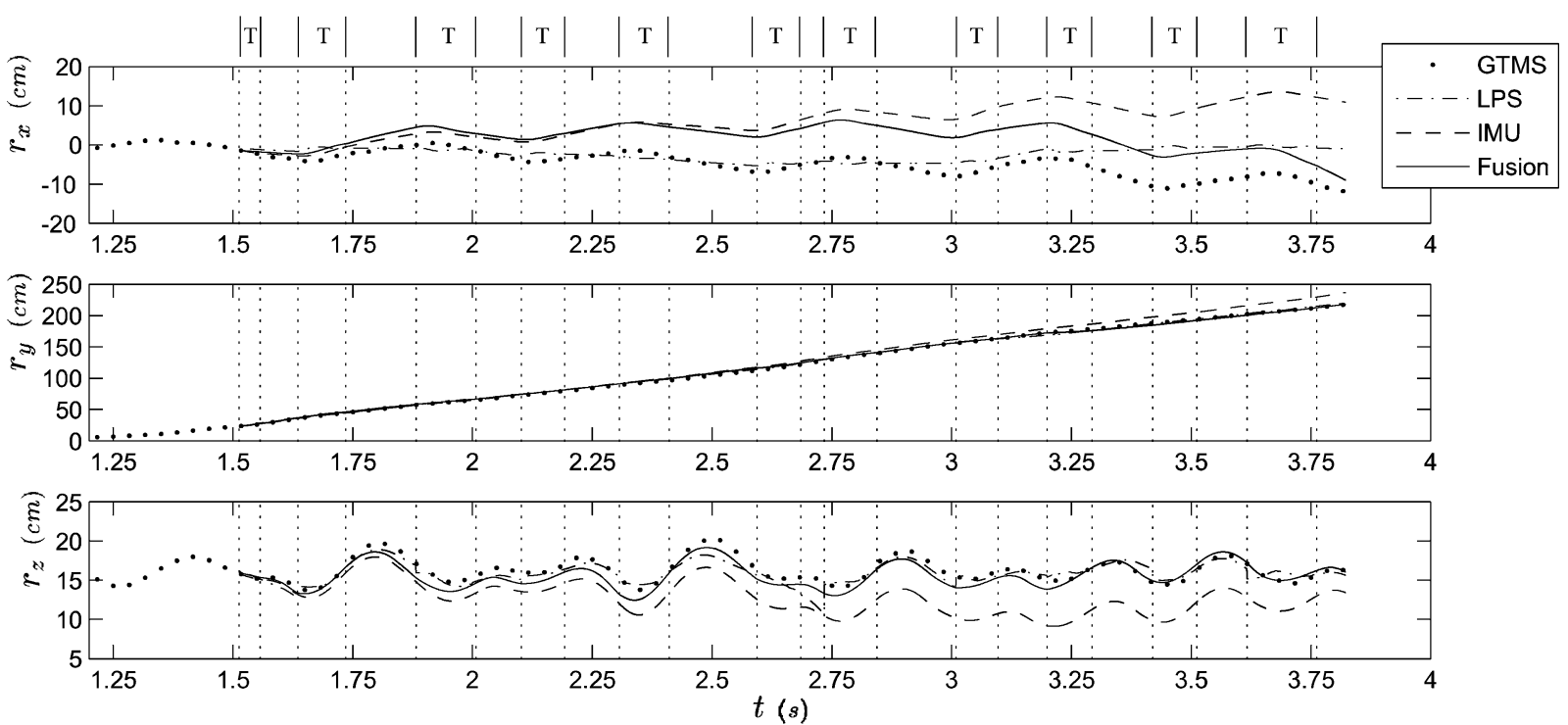

Fig. 5. Translational COM displacement [lateral $\left(r_{x}\right)$, fore/aft $\left(r_{y}\right)$, and vertical $\left(r_{z}\right)$ ] measured by GTMS (dot), LPS (a, dash-dotted line), IMU with KF (b, dashed line), and fusion algorithm (D, solid line) according to Table III. The interval $|T|$ denotes tripod stance phase determined by the leg-strain-based touchdown/ configuration sensor [12].

the LPS's cues. We believe that the interacting multiple model approach [28] holds significant promise in this context.

\section{APPENDIX I \\ ANALYSIS OF A 12-AXIS ACCELEROMETER SUITE}

\section{A. Angular Velocity From 12-Axis Accelerometer Suite}

The process to obtain an invertible mapping for this quadratic system from $\omega \times \omega$ to $\omega$ cannot be solved kinematically by the six available equations alone, since there exists at least one sign ambiguity for the unknowns. However, this is feasible, given the initial condition along with the availability of angular acceleration derived from the other six equations in the same 12-axis accelerometer suite. Without loss of generality, assuming at time $t_{i}$ the state of angular acceleration and angular velocity are available, the procedure to solve angular velocity at $t_{i+1}$ is as follows. First, derive "estimated" angular velocity by angular velocity and angular acceleration at $t_{i}$ using a constant acceleration model. Second, adopt the sign of this estimated angular velocity as the correct sign identification for angular velocity at $t_{i+1}$. Finally, choose three scalar components from solved $q(\omega)$, either $\omega_{i} \omega_{j}$ or $\omega_{i}^{2}+\omega_{j}^{2}$, where $i<j, i, j=\{1,2,3\}$, to solve angular velocity $(\omega)$, or combine both to construct a complete square $\left(\omega_{i}^{2}+\omega_{j}^{2}\right)+2 \omega_{i} \omega_{j}=\left(\omega_{i}+\omega_{j}\right)^{2}$ and solve the "fused" angular velocity.

\section{B. Structure Matrix $\mathbf{C}(d)$}

Without loss of generality, consider the shape of the robot body as a "rectangular prism" and the availability of space inside it. Numerical exploration reveals the condition number of the structure matrix $\mathbf{C}(d)$ is minimized when four 3-axis accelerometer packages occupy four of eight corners of this prism with specific and equal distances, as well as when the geometrical center of the prism coincides with the COM, as shown in Fig. 6.

The "best" condition number can be achieved in RHex's body size $(25 \mathrm{~cm} \times 50 \mathrm{~cm} \times 15 \mathrm{~cm})$ is 13.33 . However, the practical implementation of the accelerometer suite within the limited available space left in RHex, as well as having been designed to RHex to keep COM low (does not matching geometrical center) for stability, yields the high condition number of 41 in the current setup. From singular value decomposition of the structure matrix $(\mathbf{C}(d))$, we observe that the subspaces spanned by small singular values are those that effectively span the last four elements on unknown vector $\mathbf{x}_{\mathrm{var}}$ in (3), resulting in deteriorating the estimation of angular velocity. Therefore, we adopt the gyro's output as the only data source of angular velocity $\left(\omega_{g}\right)$.

\section{Short Discussion}

Since in current practical implementation, we need the extra 3-axis gyro to provide angular velocity data due to an ill-struc- 


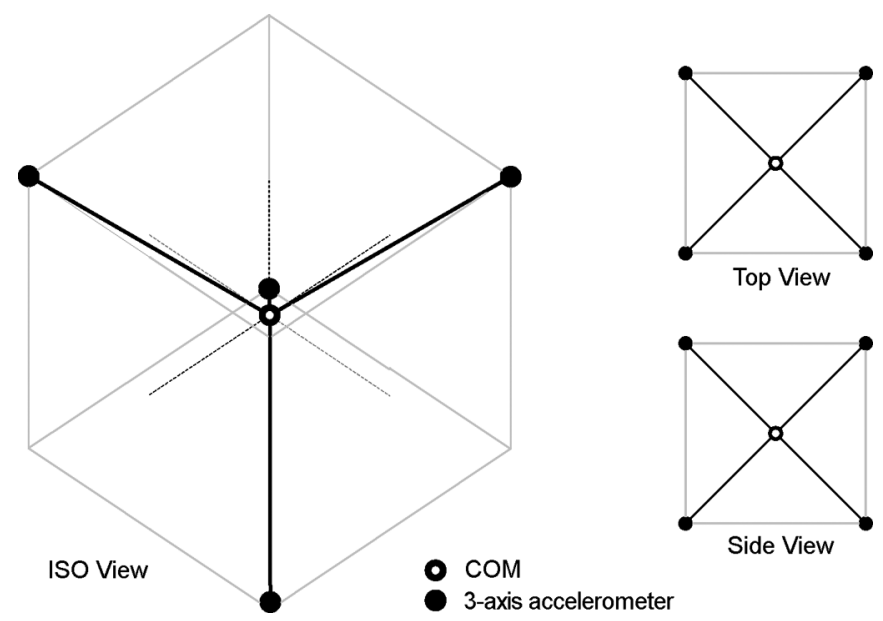

Fig. 6. Ideal locations of four 3-axis accelerometer suites with respect to the COM.

tured matrix, we attempt an alternate method of applying 6-axis accelerometer suite data and 3-axis gyro data into the right-hand side of rearranged dynamic equation (2)

$$
\mathbf{a}_{p}-\omega \times\left(\omega \times \mathbf{r}_{o p}\right)=\mathbf{a}_{o}+\dot{\omega} \times \mathbf{r}_{o p}
$$

and to solve linear and angular acceleration on the left-hand side by constructing a new $6 \times 6$ "structure" matrix $\mathbf{C}^{\prime}\left(d^{\prime}\right)$, where $d^{\prime}:=\left[\begin{array}{ll}d_{1}^{T} & d_{2}^{T}\end{array}\right] \in R^{6}$ and operate the matrix inverse. This new structure matrix $\mathbf{C}^{\prime}\left(d^{\prime}\right)$ is only nonsingular if the 6-axis acceleration is measured from at least three locations. However, this indicates that the coupled calibration detailed in Appendix II, used to compensate the misaligned installation error, is not feasible unless all locations have 3 -axis acceleration measures (at least 9 -axis total). In this situation, we prefer to install yet another 3 -axis acceleration measure (12 total) to let the computation of linear/angular acceleration remain independent of gyro measurement. We can now treat angular acceleration and angular velocity as two independent sensing data to incorporate into the EKF, detailed in Section IV-A.

\section{APPENDIX II \\ COUPLED CALIBRATION}

Theoretically, acceleration along a specific direction (usually the principal axis of the body frame; ex: $a_{z}$ ) can be obtained by a 1 -axis accelerometer $\left(\tau_{3}^{\prime}\right)$ installed on the body frame with known body orientation $(\alpha, \beta, \gamma)$, as shown in Fig. 7, left. From the reverse point of view, the sensor reading $\left(\tau_{3}^{\prime}\right)$ depends on body orientation and acceleration of that specific direction only (ex: $a_{z}$ ). However, in practical implementation, the misalignment between desired and measured directions due to installation error causes the sensor reading $\left(\tau_{i}\right)$ to be dependent on the acceleration along all three principal axes shown in Fig. 7, left: $\tau_{i}=g_{i}\left(a_{x}, a_{y}, a_{z}, \alpha, \beta, \gamma\right)_{i=1,2,3}$. For this reason, in the general case, a 3-DOF acceleration reading is required $\left(\tau_{i}, i=1,2,3\right)$ for a single acceleration along a specific direction (ex: $a_{z}$ ). If only one accelerometer measurement is applied, the acceleration measurement
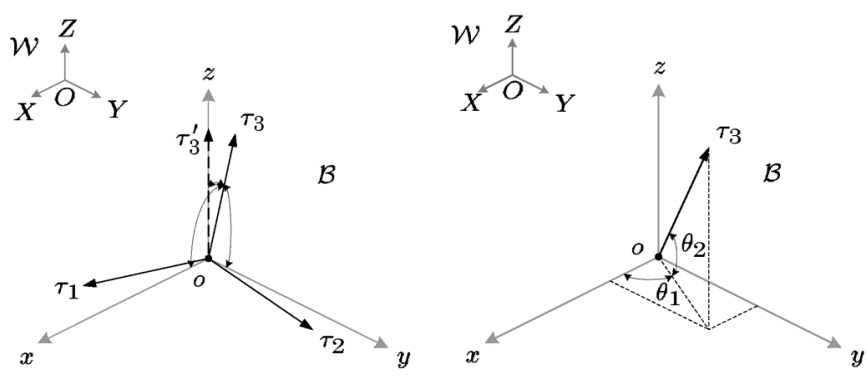

Fig. 7. Left: sketch of misalignment between accelerometer measuring directions $\left(\tau_{1}, \tau_{2}, \tau_{3}\right)$ and principal axes. Right: sketch of acceleration measure along $z$ by a misaligned accelerometer $\tau_{3}$.

in the specified direction will be contaminated by the acceleration in the remaining two directions. This practical concern motivates us to use a 3-axis accelerometer suite as the basic unit for acceleration measurement, which allows us to construct a 3-DOF coupled mapping from sensor space to orthogonal acceleration space along three principal axes $\left(\mathbf{a}_{k}\right)$ with known body orientation, expressed in the general form $\mathbf{a}_{k}=\left[\begin{array}{lll}a_{x} & a_{y} & z_{z}\end{array}\right]^{T}=g_{\text {ortho }}\left(\tau_{1}, \tau_{2}, \tau_{3}, \alpha, \beta, \gamma\right)$.

Considering the case that acceleration $\left(a_{z}\right)$ is measured by only one misaligned 1 -axis accelerometer $\left(\tau_{3}\right)$ pointing $\left(\cos \theta_{2} \cos \theta_{1}, \cos \theta_{2} \sin \theta_{1}, \sin \theta_{2}\right)$ shown in Fig. 7, right, the percentage error (\%) of measuring $a_{z}$ is given by $\left(\left(\left(a_{x} \cos \theta_{2} \cos \theta_{1}+a_{y} \cos \theta_{2} \sin \theta_{1}+a_{z} \sin \theta_{2}\right)-a_{z}\right) / a_{z}\right) \times$ 100. In the case of $\theta_{1}=45^{\circ}, \theta_{2}=85^{\circ}$ (only $5^{\circ}$ misalignment) and $a_{x}=a_{y}=a_{z}$ (acceleration has the same range along with three orthogonal components), the percentage error is $11.9 \%$. The error will increase greatly when the range of acceleration due to motion is much less than that due to gravity. For example, if $\theta_{1}=45^{\circ}, \theta_{2}=85^{\circ}$ (the same, only $5^{\circ}$ misalignment) and $a_{x}=5 a_{y}=5 a_{z}$ (assume gravity is along the direction of $a_{x}$ and the range of acceleration due to motion is only $20 \%$ of gravity), then the percentage error goes up to $36.5 \%$.

\section{ACKNOWLEDGMENT}

The authors would like to thank S. Skaff and A. Rizzi for several useful discussions. They thank G. Sharp for his GTMS implementation. Also, they thank J. Weingarten and J. Raisanen for jogging gait tuning.

\section{REFERENCES}

[1] M. Buehler, U. Saranli, and D. E. Koditschek, "Single actuator per leg robotic hexapod," U.S. Patent 6481 513, 2002, McGill Univ., Regents of the Univ. Michigan.

[2] U. Saranli, M. Buehler, and D. E. Koditschek, "Rhex-A simple and highly mobile hexapod robot," Int. J. Robot. Res., vol. 20, no. 7, pp. 616-631, 2001.

[3] J. D. Weingarten, G. A. Lopes, and D. E. Koditschek, "Automated gait generation and optimization for legged robots," in Proc. IEEE Int. Conf. Robot. Autom., 2004, vol. 3, pp. 2153-2158.

[4] E. Z. Moore and M. Buehler, "Stable stair climbing in a simple hexapod," in 4th Int. Conf. Climbing, Walking Robots, 2001, pp. 603-610.

[5] D. Campbell and M. Buehler, "Stair descent in the simple hexapod 'RHex'," in Proc. IEEE Int. Conf. Robot. Autom., 2003, vol. 1, pp. $1380-1385$.

[6] H. Komsuoḡlu, D. McMordie, U. Saranli, N. Moore, M. Buehler, and D. E. Koditschek, "Proprioception based behavioral advances in a hexapod robot," in Proc. IEEE Int. Conf. Robot. Autom., Seoul, Korea, 2001, vol. 4, pp. 3650-3655. 
[7] J. D. Weingarten, R. E. Groff, and D. E. Koditschek, "A framework for the coordination of legged robot gaits," in Proc. IEEE Int. Conf. Robot., Autom., Mechatron., 2004, pp. 679-686.

[8] U. Saranli, A. Rizzi, and D. Koditschek, "Model-based dynamic selfrighting maneuvers for a hexapedal robot," Int. J. Robot. Res., vol. 23, no. 9, pp. 903-918, 2004.

[9] N. Neville and M. Buehler, "Towards bipedal running of a six legged robot," in Proc. 12th Yale Workshop Adaptive, Learning Syst., May 2003.

[10] D. McMordie and M. Buehler, "Towards pronking with a hexapod robot," in Proc. 4th Int. Conf. Climbing, Walking Robots, 2001, pp. 659-666.

[11] U. Saranli, "Dynamic locomotion in a hexapod robot," Ph.D. dissertation, Univ. Michigan, Ann Arbor, Aug. 2002.

[12] P. Lin, H. Komsuoḡlu, and D. E. Koditschek, "A leg configuration measurement system for full body pose estimates in a hexapod robot," IEEE Trans. Robot., vol. 21, no. 3, pp. 411-422, Jun., 2005.

[13] R. Altendorfer, N. Moore, H. Komsuoḡlu, H. B. Brown, D. McMordie, U. Saranli, R. Full, and D. E. Koditschek, "RHex: A biologically inspired hexapod runner," Auton. Robots, vol. 11, no. 3, pp. 207-213, 2001.

[14] P. Lin, H. Komsuoḡlu, and D. E. Koditschek, "Toward a 6-DOF body state estimator for a hexapod robot with dynamical gaits," in Proc. IEEE/RSJ Int. Conf. Intell. Robots Syst., 2004, pp. 2265-2270.

[15] B. Barshan and H. F. Durrant-Whyte, "Inertial navigation systems for mobile robots," IEEE Trans. Robot. Autom., vol. 11, no. 3, pp. 328-342, Jun. 1995.

[16] A. J. Padgaonkar, K. W. Krieger, and A. I. King, "Measurement of angular accelerarion of a rigid body using linear accelerometers," Trans. ASME, vol. 42, pp. 552-556, 1975.

[17] P. J. Escamilla-Ambrosio and N. Mort, "A hybrid Kalman filter-fuzzy logic architecture for multisensor data fusion," in Proc. Int. Symp. Intell. Control, 2001, pp. 364-369.

[18] M. Abdelrahman and P. Abdelrahman, "Integration of multiple sensor fusion in controlled design," in Proc. Amer. Control Conf., 2002, pp. 2609-2614.

[19] S. Sukkarieh, E. M. Nebot, and H. F. Durrant-Whyte, "A high-integrity IMU/GPS navigation loop for autonomous land vehicle application," IEEE Trans. Robot. Autom., vol. 15, no. 3, pp. 572-578, Jun. 1999.

[20] G. Dissanayaka, S. Sukkarieh, E. M. Nebot, and H. F. Durrant-Whyte, "The aiding of a low-cost strapdown inertial measurement unit using vehicle model constraints for land vehicle applications," IEEE Trans. Robot. Autom., vol. 17, no. 5, pp. 731-747, Oct. 2001.

[21] S. I. Roumeliotis, A. E. Johnson, and J. F. Montgomery, "Augmenting inertial navigation with image-based motion estimation," in Proc. IEEE Int. Conf. Robot. Autom., 2002, vol. 4, pp. 4326-4333.

[22] O. Wijk and H. I. Christensen, "Triangulation-based fusion of sonar data with application in robot pose tracking," IEEE Trans. Robot. Autom., vol. 16, no. 6, pp. 740-752, Dec. 2000 .

[23] R. C. Ren, S. H. Phang, and K. L. Su, "Multilevel multisensor based decision fusion for intelligent animal robot," in Proc. IEEE Int. Conf. Robot. Autom., 2001, vol. 4, pp. 4226-4231.

[24] G. C. Sharp, Univ. Michigan, "Ground Truth Measurement System." (2003) [Online]. Available: http://www.sourceforge.net/projects/gtms/

[25] P. Lin, "Proprioceptive sensing for a legged robot," Ph.D. dissertation, Univ. Michigan, Ann Arbor, Aug. 2005.

[26] U. Saranli and D. E. Koditschek, "Template based control of hexapedal running," in Proc. IEEE Int. Conf. Robot. Autom., 2003, vol. 1, pp. 1374-1379.
[27] R. Altendorfer, D. E. Koditschek, and P. Holmes, "Stability analysis of a clock-driven rigid-body slip model for RHex," Int. J. Robot. Res. vol. 23, no. 11, pp. 1001-1012, 2004.

[28] S. Skaff, A. Rizzi, H. Choset, and P. Lin, "Context-based state estimation technique for hybrid systems," in Proc. IEEE Int. Conf. Robot. Autom., Apr. 2005, pp. 3935-3940.

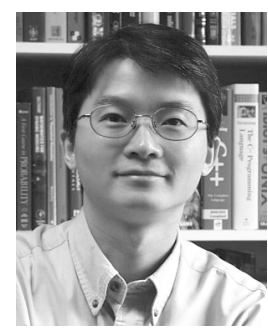

Pei-Chun Lin (S'02-M'05) received the B.S. and M.S. degrees in mechanical engineering from National Taiwan University (NTU), Taipei, Taiwan, R.O.C., in 1996 and 1998, respectively, and the M.S. degree in electrical engineering and computer science and the Ph.D. degree in mechanical engineering from The University of Michigan, Ann Arbor, in 2005.

Currently, he is a Postdoctoral Research Fellow with the University of Pennsylvania, Philadelphia. His research interests include mechanical design, sensor design, sensor fusion, and analysis of robot dynamic behaviors.

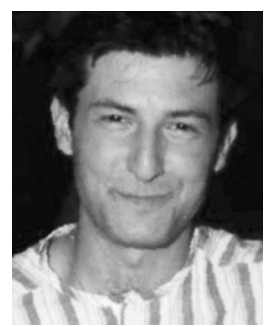

Haldun Komsuoḡlu (S'95-M'04) received the B.S. degree from the Department of Electrical and Electronics Engineering, Middle East Technical University (METU), Ankara, Turkey, in 1997. He received the M.S. degree in 1998 with a controls major and the Ph.D. degree in 2004, with a dissertation entitled "Toward a formal framework for open-loop control of rhythmic tasks," from the Department of Electrical Engineering and Computer Science, University of Michigan, Ann Arbor.

His research interests include open-loop control of hybrid dynamical systems, design and characterization of self-stabilizing mechanisms with applications in legged locomotion, as well as design of embedded sensory and actuation systems for robotic platforms.

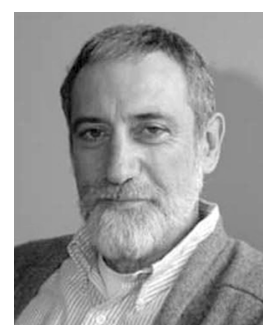

Daniel E. Koditschek (S'80-M'83-SM'93-F'04) received the $\mathrm{Ph} . \mathrm{D}$. degree in electrical engineering from Yale University, New Haven, CT, in 1983.

He served on the faculty of the Department of Electrical Engineering, Yale University, for 10 years, and then joined the Department of Electrical Engineering and Computer Science, University of Michigan, Ann Arbor, in 1993. He joined the University of Pennsylvania, Philadelphia, in January 2005 , where he holds secondary appointments with the Departments of Computer and Information Science and Mechanical Engineering and Applied Mechanics. He is also the Alfred Fitler Moore Professor and Chairman of Electrical and Systems Engineering at the School of Engineering and Applied Science. His research interests include robotics, the application of dynamical systems theory to intelligent machines, and nonlinear control. 